\title{
Free vibration analysis of thin circular and annular plate with general boundary conditions
}

\author{
S. Khare* and N. D. Mittal
}

Department of Mechanical Engineering, Mulana Azad National Institute of Technology, Bhopal, India

\begin{tabular}{|c|c|}
\hline ARTICLE INFO & ABSTRACT \\
\hline $\begin{array}{l}\text { Article history: } \\
\text { Received } 6 \text { April, } 2015 \\
\text { Accepted 12 June } 2015 \\
\text { Available online } \\
\text { 14 June } 2015 \\
\text { Keywords: } \\
\text { Circular and annular plate } \\
\text { Natural frequency } \\
\text { Mode shape } \\
\text { Finite element method }\end{array}$ & $\begin{array}{l}\text { This paper presents a numerical analysis of free vibration of thin circular and annular plate } \\
\text { using finite element method. The first five natural frequencies are presented for uniform } \\
\text { annular plates of various inner-to-outer radius ratios, with nine possible combinations of free, } \\
\text { clamped and simply supported boundary conditions at the inner and outer edges of plates. The } \\
\text { accuracy of the method is established by comparing the results available in the literature. } \\
\text { Results show that natural frequency parameter increases as the inner-to-outer radius ratio } \\
\text { increases except in case of free boundary condition, for which it decreases with the inner-to- } \\
\text { outer radius ratio. This result provides benchmark values that can be used to validate result } \\
\text { obtained by other approximate approaches such as finite difference method, differential } \\
\text { quadrature method and boundary element method. }\end{array}$ \\
\hline
\end{tabular}

(C) 2015 Growing Science Ltd. All rights reserved.

\section{Introduction}

Circular and annular plates are the fundamental structural elements used in various engineering fields. The lateral vibration of such plates has been the subject of numerous studies. Yuan and Dickinson (1996) studied the natural frequency parameters for the free vibration of annular, circular and sectorial plates using a Ritz solution. Vera et al. $(1998,1999)$ studied free vibration of annular plates with four combinations of boundary conditions Case (i) clamped at both edges, Case (ii) clamped at outer edge and simply supported at inner edge, Case (iii) simply supported at outer edge and clamped at inner edge and Case (iv) simply supported at both edges and also the free-free edge conditions.

Chakraverty and Petyt (1999) studied elliptical and circular plates with seven types of orthotropic material properties for all the classical free, simply supported and clamped boundary conditions using the Rayleigh-Ritz method with two-dimensional boundary characteristic orthogonal polynomials as the admissible functions. They presented an exhaustive graphical result of the first five frequencies for various aspect ratios. Chakraverty et al. $(2000,2001)$ also studied the orthotropic annular elliptic plates.

* Corresponding author.

E-mail addresses: sumitkhare8686@gmail.com (S. Khare) 
Their study contains results for the first eight frequency parameters for various values of aspect ratios of the outer and inner ellipse. Wu et al. (2002) studied the free vibration of solid circular plates using the generalized differential quadrature rule (GDQR). Kim (2003) analyzed natural frequency parameters for isotropic elliptical and circular plates using the Rayleigh-Ritz method. Farag and Pan (2003) used the assumed method to thoroughly investigate the in-plane modal characteristics of a solid circular disk with clamped outer boundary.

Wang (2003) studied the vibration of an annular membrane attached to a free rigid core. Wang et al. (2005) studied the effect of core on the fundamental frequencies of annular plates with four different types of ideal boundary conditions. Bashmal et al. (2009) used the Rayleigh-Ritz method with boundary characteristic orthogonal polynomials (BCOP) to study the free in-plane vibrations of annular plates for different combinations of inner/outer edge conditions. The latter authors subsequently derived the exact frequency equations, based on the 2D linear plane stress theory, in terms of Bessel functions (2010). Hassani et al. (2010) employed a Rayleigh-Ritz approach with two-dimensional BCOP and linear plane stress theory to investigate the in-plane modal characteristics of annular circular and elliptic plates of non-uniform thickness for all classical boundary conditions.

Komur et al. (2010) carried out a buckling analysis for laminated composite plates with an elliptical/circular hole centered in the plate using finite element method (FEM) using ANSYS finite element software. Chen and Ren (1998) studied the lateral vibration of thin annular and circular plates with variable thickness using finite element analysis. Liang et al. (2007) extended a new method using the limited finite element method (FEM) for the analysis of the natural frequencies of circular/annular plates of polar orthotropy, stepped and variable thickness. Moreover, free vibration analysis of different geometries and materials have also been studied numericaly or theoretically in recent years (Torabi et al. 2013; Nia et al. 2014; Vimal et al. 2014; Yadav et al. 2015; Bhardwaj et al. 2015; Samaei et al. 2015). In this paper, the effects of different radii ratio, with nine possible combinations of free, clamped and simply supported boundary conditions at the inner and outer edges of plates on the free vibration responses are discussed in detail.

\section{Material and Methods}

A finite element analysis was made for obtaining the first five natural frequencies using ANSYS. The free vibration is computed using Block-Lanczos algorithm. In addition SHELL 181 is suitable for analyzing thin to moderately-thick shell structures. As demonstrated in Fig. 1, the element contains four-node with six degree of freedom at each node. SHELL 181 is well suited for linear, large rotation, and/or large strain nonlinear applications.

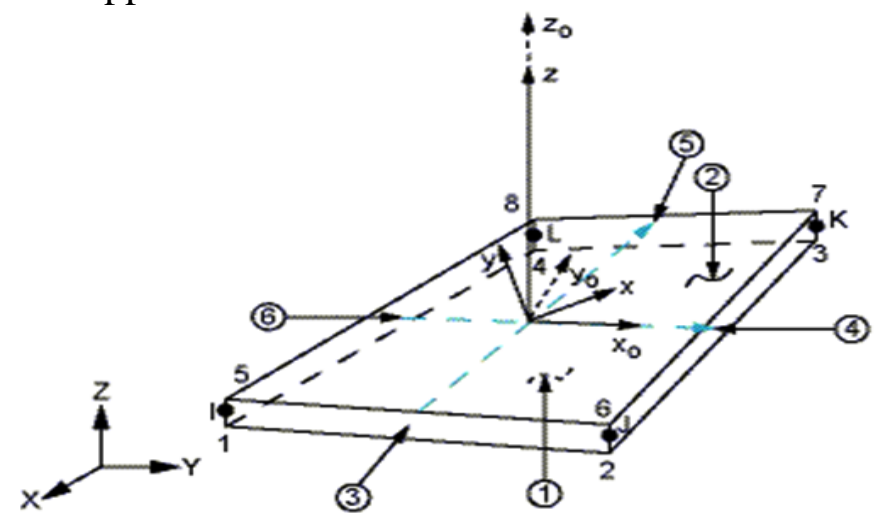

Fig. 1. Four noded SHELL 181 element 
Consider an isotropic, homogeneous annular plate with uniform thickness $h$ in cylindrical coordinate $(r, \theta, z)$ with the z-axis along the longitudinal direction $R_{\mathbf{1}}$ and $R_{2}$ are the inner radius and outer radius as shown in Fig. 2.

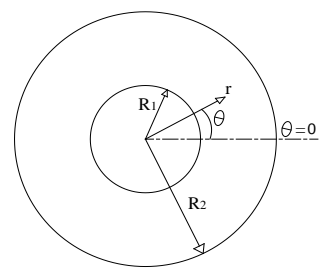

Fig. 2. Geometry and coordinate system of the annular plate
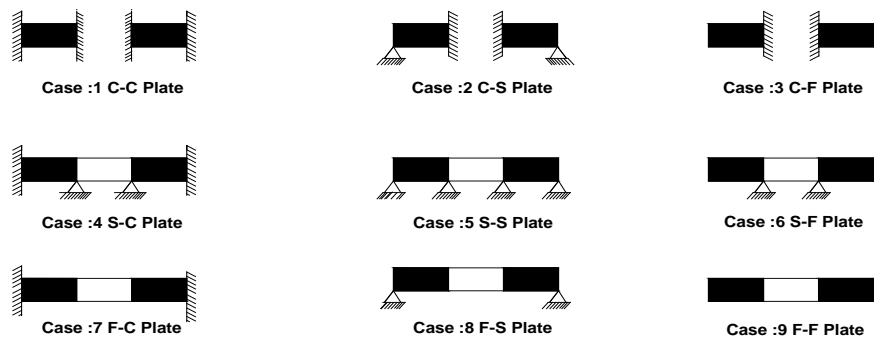

Fig. 3. The boundary conditions of the annular plates analyzed

In this study, isotropic plates made of steel were used. The thickness of plate remains uniform throughout the study. The mechanical properties of steel are listed in Table 1.

Table 1. Mechanical properties of the steel (Reddy 2004)

\begin{tabular}{llllll}
\hline $\mathrm{E}_{1}$ & $\mathrm{E}_{2}$ & $\mathrm{G}_{12}$ & $\mathrm{G}_{13}$ & $\mathrm{G}_{23}$ & $\mu$ \\
\hline 30 & 30 & 11.24 & 11.24 & 11.24 & 0.29 \\
\hline
\end{tabular}

Moduli are in msi=million psi ; 1 psi $=6.895 \mathrm{kN} / \mathrm{m}^{2}$

\section{Results and discussion}

\subsection{Circular plate}

The present study is first validated by carrying out convergence study of non-dimensional frequency parameter $\Omega$ defined by $\Omega=\omega \mathrm{R}_{2} \sqrt{\rho h / D}$, with respect to mesh dimensions $(\mathrm{M} \times \mathrm{N})$ and by comparison with results available in the literature. The rates of convergence of the first five frequency parameter for free, clamped and simply supported boundary conditions are presented in Tables 2 to 4 . It can be seen that $\mathrm{M}=12, \mathrm{~N}=12$, is sufficient for converged result. The first five natural modes of flexural vibrations for free, clamped and simply plates are shown in Fig. 4. It can be noted that for free plate mode $(1,0)$ has higher frequency than mode $(1,2)$, as well as mode $(1,1)$ than $(1,3)$.

Table 2. Value of frequency parameter $\Omega=\omega \mathrm{R}_{2} \sqrt{\rho h / D}$ for circular plate with clamped boundary condition.

\begin{tabular}{cccccc}
\hline NxM & \multicolumn{5}{c}{ Mode Number } \\
\cline { 2 - 6 } & 1 & 2 & 3 & 4 & 5 \\
\hline 6 x6 & 10.424 & 22.443 & 38.613 & 48.544 & 58.704 \\
7x7 & 10.310 & 22.191 & 37.554 & 45.416 & 57.600 \\
8x8 & 10.293 & 21.772 & 36.900 & 45.104 & 55.240 \\
9x9 & 10.253 & 21.78 & 36.318 & 43.808 & 54.116 \\
10x10 & 10.192 & 21.408 & 35.504 & 42.636 & 52.976 \\
\hline $11 \times 11$ & 10.186 & 21.396 & 35.469 & 42.100 & 52.364 \\
\hline 12x12 & 10.148 & 21.454 & 35.694 & 41.136 & 52.952 \\
\hline Chakraverty et al. (2001) & 10.220 & 21.260 & 34.880 & 39.770 & 51.030 \\
\hline & 10.2158 & 21.260 & 34.877 & 39.7711 & 51.0306 \\
\hline
\end{tabular}


Table 3. Value of frequency parameter $\Omega=\omega \mathrm{R}_{2} \sqrt{\rho h / D}$ for circular plate with simply supported boundary condition

\begin{tabular}{cccccc}
\hline \multirow{2}{*}{ NxM } & \multicolumn{5}{c}{ Mode Number } \\
\cline { 2 - 6 } & 1 & 2 & 3 & 4 & 5 \\
\hline $6 \times 6$ & 4.9228 & 14.31 & 27.297 & 34.354 & 44.16 \\
$7 \times 7$ & 4.9028 & 14.216 & 26.823 & 32.656 & 43.364 \\
$8 \times 8$ & 4.8936 & 14.002 & 26.411 & 32.51 & 42.176 \\
$9 \times 9$ & 4.8852 & 13.984 & 26.138 & 31.807 & 41.38 \\
$10 \times 10$ & 4.8696 & 13.842 & 25.718 & 31.127 & 40.812 \\
$11 \times 11$ & 4.8708 & 13.83 & 25.66 & 30.846 & 40.392 \\
$12 \times 12$ & 4.8664 & 13.864 & 25.792 & 30.285 & 40.7 \\
\hline Chakraverty et al. (2001) & 4.984 & 13.94 & 25.65 & 29.76 & $34.00^{*}$ \\
Zhou et al. (2011) & 4.9352 & 13.8983 & 25.6148 & 29.7193 & 39.9574 \\
\hline
\end{tabular}

Table 4. Value of frequency parameter $\Omega=\omega \mathrm{R}_{2} \sqrt{\rho h / D}$ for circular plate with free boundary condition

\begin{tabular}{cccccc}
\hline \multirow{2}{*}{ NxM } & \multicolumn{5}{c}{ Mode Number } \\
\cline { 2 - 6 } & 1 & 2 & 3 & 4 & 5 \\
\hline $6 \times 6$ & 5.4088 & 9.2032 & 12.911 & 21.484 & 23.684 \\
$7 \times 7$ & 5.3628 & 9.0912 & 12.691 & 21.242 & 23.009 \\
$8 \times 8$ & 5.3408 & 9.0768 & 12.592 & 20.867 & 22.643 \\
$9 \times 9$ & 5.32 & 9.0404 & 12.515 & 20.833 & 22.358 \\
$10 \times 10$ & 5.3132 & 8.9792 & 12.467 & 20.547 & 22.232 \\
$11 \times 11$ & 5.298 & 8.9688 & 12.408 & 20.556 & 22.092 \\
$12 \times 12$ & 5.2936 & 8.9288 & 12.38 & 20.587 & 21.985 \\
\hline Chakravertv et al. (2001) & 5.251 & 9.076 & 12.22 & 20.52 & 21.49 \\
Zhou et al. (2011) & 5.3583 & 9.0031 & 12.439 & 20.4745 & 21.8352 \\
\hline
\end{tabular}

$\mathrm{C}$

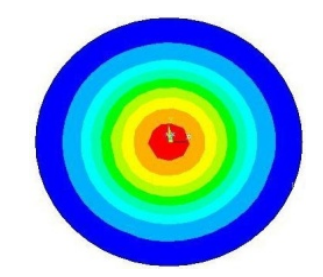

Mode $1(1,0)$

$\mathrm{F}$

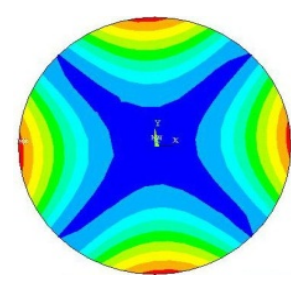

Mode $1(1,2)$

SS

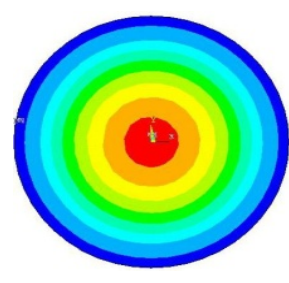

Mode $1(1,0)$

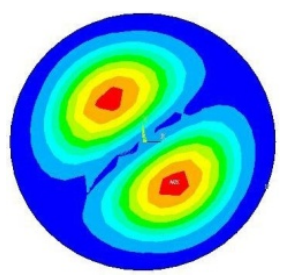

Mode 2(1, 1)

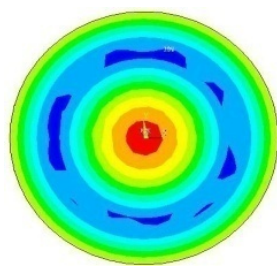

Mode $2(1,0)$

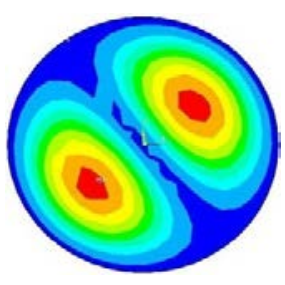

Mode 2(1, 1)

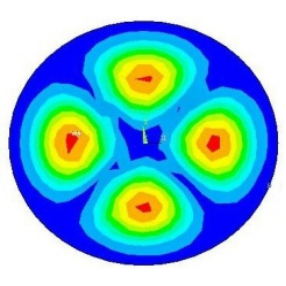

Mode 3(1, 2)

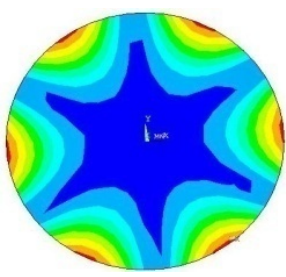

Mode 3 (1, 3)

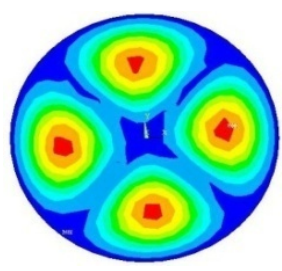

Mode 3 (1, 2)

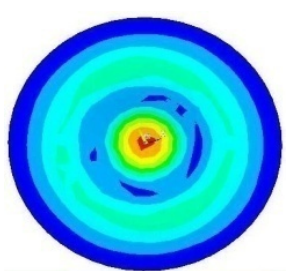

Mode 4(2, 0)

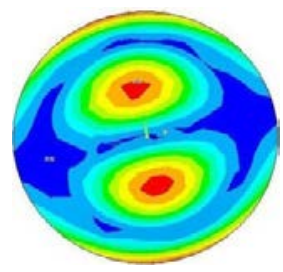

Mode $4(1,1)$

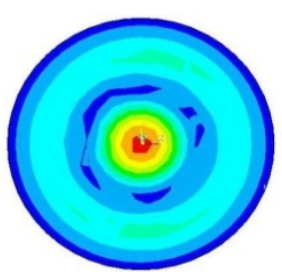

Mode $4(2,0)$

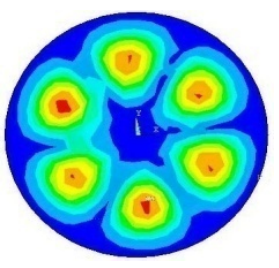

Mode 5(1, 3)

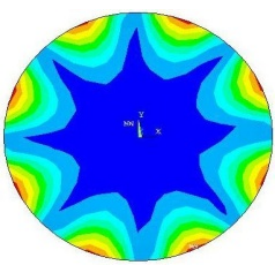

Mode 5 (1, 4)

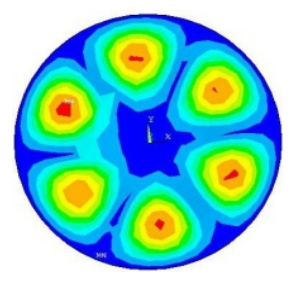

Mode $5(1,3)$

Fig. 4. The first five natural modes of clamped (C), free (F) and simply supported (SS) circular plate 


\subsection{Annular Plate}

The first five non-dimensional frequency parameters, for uniform annular plates of various innerto-outer radius ratios varying from 0.1 to 0.8 at interval of 0.1 are computed and presented in Tables 513. Result are provided for nine cases of boundary conditions (C-S, C-F; S-C, S-S, S-F; F-C, F-S, F-F) at both the inner and outer edges of plates (Fig. 3). Here, the designation C-S identifies a plate with the outer edge clamped and the inner edge simply supported and F-C corresponds to a free outer edge.

Table 5. Value of frequency parameter $\Omega$ for C-C plate

\begin{tabular}{cccccc}
\hline \multicolumn{5}{c}{ Mode number } \\
$\mathrm{R}_{1} / \mathrm{R}_{2}$ & 1 & 2 & 3 & 4 & 5 \\
\hline 0.1 & 27.252 & 28.882 & 36.607 & 51.328 & 70.044 \\
0.2 & 34.613 & 36.109 & 41.828 & 53.448 & 70.468 \\
0.3 & 45.48 & 46.776 & 51.26 & 60.196 & 74.236 \\
0.4 & 61.864 & 62.948 & 66.552 & 73.42 & 84.372 \\
0.5 & 89.62 & 90.592 & 93.66 & 99.268 & 107.9 \\
0.6 & 139.596 & 140.452 & 143.088 & 147.736 & 154.664 \\
0.7 & 248.528 & 249.28 & 251.576 & 255.536 & 261.268 \\
0.8 & 559.16 & 559.8 & 561.84 & 565.32 & 570.16 \\
\hline
\end{tabular}

Table 7. Value of frequency parameter $\Omega$ for C-S plate

\begin{tabular}{crrrrr}
\hline \multicolumn{5}{c}{ Mode number } \\
$\mathrm{R}_{1} / \mathrm{R}_{2}$ & 1 & \multicolumn{1}{c}{2} & \multicolumn{1}{c}{3} & \multicolumn{1}{c}{4} & \multicolumn{1}{c}{5} \\
\hline 0.1 & 22.551 & 25.098 & 35.330 & 51.168 & 65.696 \\
0.2 & 26.595 & 29.138 & 37.567 & 51.736 & 69.996 \\
0.3 & 33.683 & 35.840 & 42.708 & 54.676 & 71.312 \\
0.4 & 44.628 & 46.400 & 51.956 & 61.700 & 75.836 \\
0.5 & 63.964 & 65.476 & 70.128 & 78.200 & 89.912 \\
0.6 & 98.640 & 99.972 & 103.884 & 110.588 & 120.248 \\
0.7 & 174.144 & 175.248 & 178.588 & 184.248 & 192.316 \\
0.8 & 389.128 & 390.08 & 392.968 & 397.824 & 404.640 \\
\hline
\end{tabular}

Table 9. Value of frequency parameter $\Omega$ for F-F plate

\begin{tabular}{ccrccc}
\hline & \multicolumn{5}{c}{ Mode number } \\
$\mathrm{R}_{1} / \mathrm{R}_{2}$ & 1 & \multicolumn{1}{c}{ } & 3 & 4 & 5 \\
\hline 0.1 & 5.1904 & 8.7956 & 12.2184 & 20.4072 & 21.5392 \\
0.2 & 5.0408 & 8.4216 & 12.1760 & 19.6464 & 21.4972 \\
0.3 & 4.8132 & 8.2964 & 12.0552 & 18.1508 & 21.4724 \\
0.4 & 4.4596 & 8.3960 & 11.6068 & 16.7792 & 21.0590 \\
0.5 & 4.1980 & 9.2044 & 11.2432 & 16.9104 & 20.7572 \\
0.6 & 3.8546 & 10.5164 & 10.5340 & 18.1472 & 19.7876 \\
0.7 & 3.5139 & 9.7088 & 12.9820 & 18.4272 & 21.4624 \\
0.8 & 3.1883 & 8.8480 & 16.8488 & 18.2100 & 27.1744 \\
\hline
\end{tabular}

Table 11. Value of frequency parameter $\Omega$ for S-C plate

\begin{tabular}{crrrrr}
\hline & \multicolumn{5}{c}{ Mode number } \\
$\mathrm{R}_{1} / \mathrm{R}_{2}$ & \multicolumn{1}{c}{1} & \multicolumn{1}{c}{3} & \multicolumn{1}{c}{3} & \multicolumn{1}{c}{5} \\
\hline 0.1 & 17.814 & 19.4124 & 26.7436 & 40.180 & 57.164 \\
0.2 & 22.7524 & 24.3084 & 30.1192 & 41.392 & 57.320 \\
0.3 & 30.076 & 31.498 & 36.330 & 45.572 & 59.492 \\
0.4 & 41.028 & 42.284 & 46.372 & 53.928 & 65.516 \\
0.5 & 60.020 & 61.180 & 64.808 & 71.276 & 80.968 \\
0.6 & 94.168 & 95.224 & 98.452 & 104.056 & 112.256 \\
0.7 & 168.560 & 169.516 & 172.424 & 177.224 & 184.488 \\
0.8 & 381.288 & 382.156 & 384.804 & 389.256 & 395.54 \\
\hline
\end{tabular}

Table 6. Value of frequency parameter $\Omega$ for C-S plate

\begin{tabular}{rrrrrr}
\hline \multicolumn{5}{c}{ Mode number } \\
$\mathrm{R}_{1} / \mathrm{R}_{2}$ & \multicolumn{1}{c}{1} & \multicolumn{1}{c}{2} & \multicolumn{1}{c}{3} & \multicolumn{1}{c}{4} & \multicolumn{1}{c}{5} \\
\hline 0.1 & 10.106 & 21.154 & 34.549 & 39.387 & 51.104 \\
0.2 & 10.319 & 20.46 & 33.751 & 42.816 & 50.576 \\
0.3 & 11.305 & 19.364 & 32.556 & 49.18 & 51.648 \\
0.4 & 13.24 & 19.612 & 30.924 & 46.408 & 65.632 \\
0.5 & 17.546 & 21.73 & 31.7224 & 45.456 & 62.8 \\
0.6 & 25.468 & 28.42 & 36.326 & 47.928 & 62.812 \\
0.7 & 42.86 & 44.952 & 50.972 & 60.416 & 72.92 \\
0.8 & 92.556 & 94.052 & 98.504 & 105.816 & 115.864 \\
\hline
\end{tabular}

Table 8. Value of frequency parameter $\Omega$ for F-C plate

\begin{tabular}{rrrrrr}
\hline \multicolumn{5}{c}{ Mode number } \\
$\mathrm{R}_{1} / \mathrm{R}_{2}$ & \multicolumn{1}{c}{1} & \multicolumn{1}{c}{3} & \multicolumn{1}{c}{3} & \multicolumn{1}{c}{4} & \multicolumn{1}{c}{5} \\
\hline 0.1 & 3.47096 & 4.2524 & 5.506 & 12.2316 & 21.5396 \\
0.2 & 4.808 & 5.2016 & 6.3324 & 12.3832 & 21.5248 \\
0.3 & 6.550 & 6.6884 & 7.8444 & 13.0412 & 21.7496 \\
0.4 & 8.926 & 8.9864 & 10.2048 & 14.5104 & 22.366 \\
0.5 & 13.0636 & 13.2884 & 14.582 & 18.2792 & 25.1664 \\
0.6 & 20.5544 & 20.9228 & 22.3584 & 25.652 & 31.540 \\
0.7 & 36.9756 & 37.472 & 39.110 & 42.276 & 47.448 \\
0.8 & 84.456 & 85.064 & 86.944 & 90.232 & 95.128 \\
\hline
\end{tabular}

Table 10. Value of frequency parameter $\Omega$ for F-S plate

\begin{tabular}{crrrrr}
\hline \multicolumn{5}{c}{ Mode number } \\
$\mathrm{R}_{1} / \mathrm{R}_{2}$ & \multicolumn{1}{c}{1} & \multicolumn{1}{c}{3} & \multicolumn{1}{c}{3} & \multicolumn{1}{c}{4} & \multicolumn{1}{c}{5} \\
\hline 0.1 & 2.3690 & 3.4316 & 5.2996 & 12.222 & 20.820 \\
0.2 & 2.8385 & 3.3052 & 5.5272 & 12.240 & 21.5048 \\
0.3 & 3.3130 & 3.379 & 5.9456 & 12.3812 & 21.5576 \\
0.4 & 3.5664 & 3.8638 & 6.5568 & 12.618 & 21.5056 \\
0.5 & 4.060 & 4.760 & 7.7936 & 13.7384 & 22.3832 \\
0.6 & 4.795 & 6.030 & 9.6940 & 15.755 & 24.2040 \\
0.7 & 6.0924 & 8.1648 & 13.080 & 19.9432 & 28.7308 \\
0.8 & 8.758 & 12.448 & 20.098 & 29.4356 & 40.1720 \\
\hline
\end{tabular}

Table 12. Value of frequency parameter $\Omega$ for S-F plate

\begin{tabular}{cccccr}
\hline \multicolumn{5}{c}{ Mode number } \\
$\mathrm{R}_{1} / \mathrm{R}_{2}$ & 1 & 2 & 3 & 4 & \multicolumn{1}{c}{5} \\
\hline 0.1 & 4.8764 & 13.8896 & 25.4364 & 29.3612 & 40.060 \\
0.2 & 4.7196 & 13.5604 & 24.9116 & 31.2440 & 39.7288 \\
0.3 & 4.6460 & 12.7552 & 24.1504 & 36.9076 & 38.8872 \\
0.4 & 4.6536 & 11.7168 & 22.7908 & 36.8832 & 36.8884 \\
0.5 & 5.0284 & 11.4416 & 22.1488 & 35.4844 & 51.988 \\
0.6 & 5.6472 & 11.6984 & 22.0988 & 34.6840 & 49.832 \\
0.7 & 6.8448 & 13.0548 & 23.8608 & 36.4640 & 50.968 \\
0.8 & 9.4284 & 16.7356 & 29.5732 & 43.9920 & 59.736 \\
\hline
\end{tabular}

Table 13. Value of frequency parameter $\Omega$ for $\mathrm{S}-\mathrm{S}$ plate

\begin{tabular}{|c|c|c|c|c|c|}
\hline \multicolumn{6}{|c|}{ Mode number } \\
\hline $\mathrm{R}_{1} / \mathrm{R}_{2}$ & 1 & 2 & 3 & 4 & 5 \\
\hline 0.1 & 14.4144 & 16.6792 & 25.9192 & 40.092 & 51.78 \\
\hline 0.2 & 16.7048 & 19.1732 & 27.2332 & 40.36 & 57.068 \\
\hline 0.3 & 21.0264 & 23.2804 & 30.2704 & 41.976 & 57.748 \\
\hline 0.4 & 27.7664 & 29.7328 & 35.734 & 45.852 & 59.988 \\
\hline 0.5 & 40.008 & 41.764 & 47.056 & 55.944 & 68.400 \\
\hline 0.6 & 62.016 & 63.568 & 68.224 & 76.012 & 86.932 \\
\hline 0.7 & 109.872 & 111.248 & 115.38 & 122.28 & 131.94 \\
\hline 0.8 & 247.0716 & 248.28 & 251.996 & 258.158 & 266.790 \\
\hline
\end{tabular}


C-C
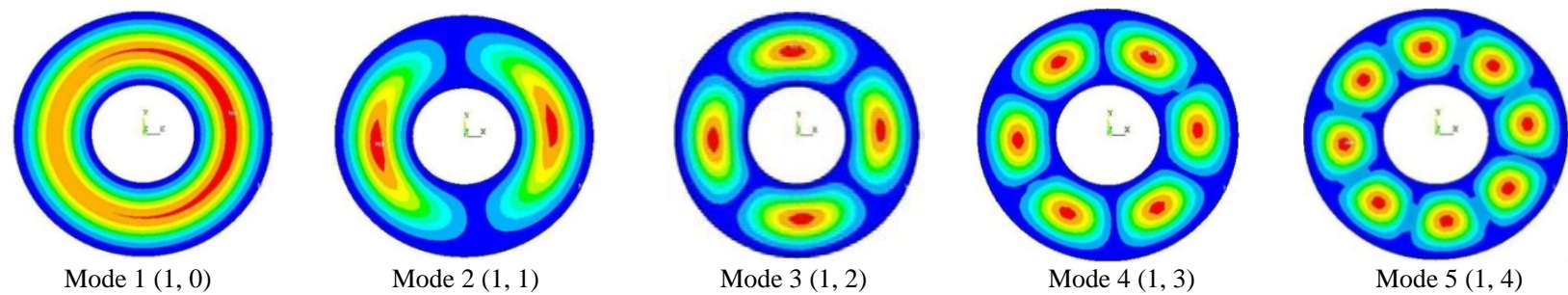

C-F
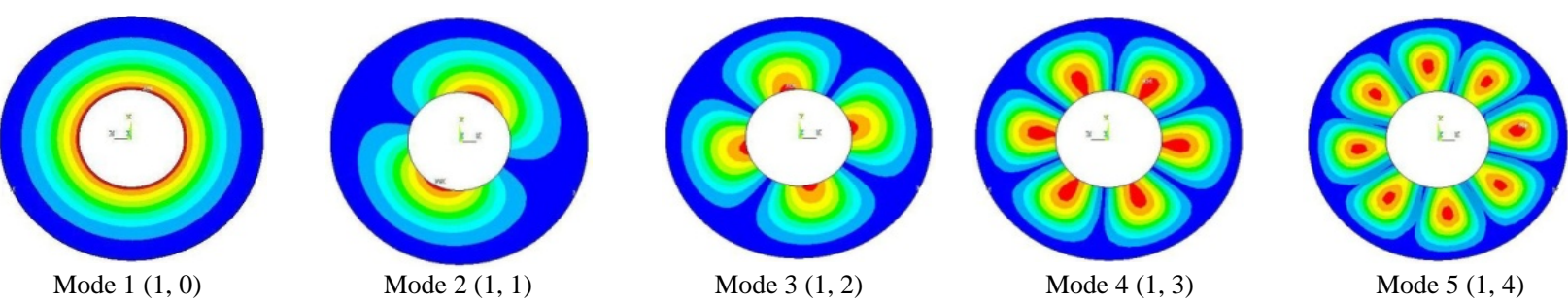

C-S
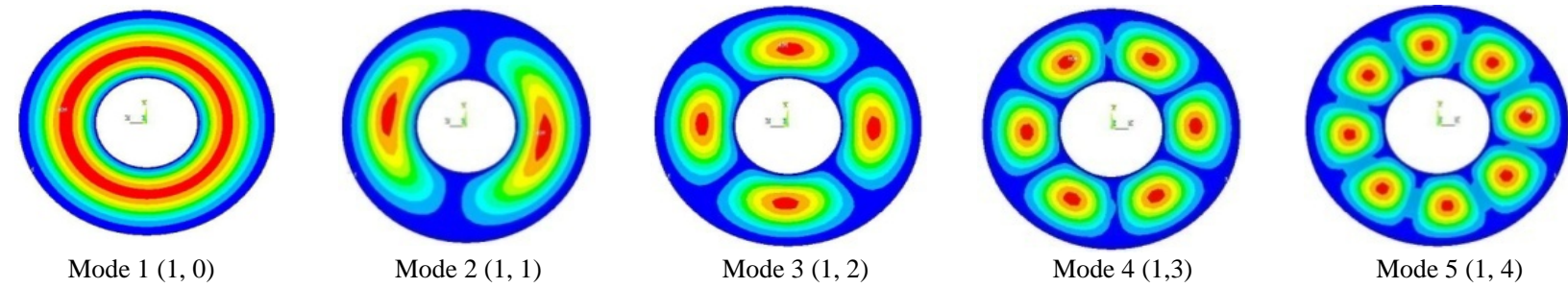

F.C
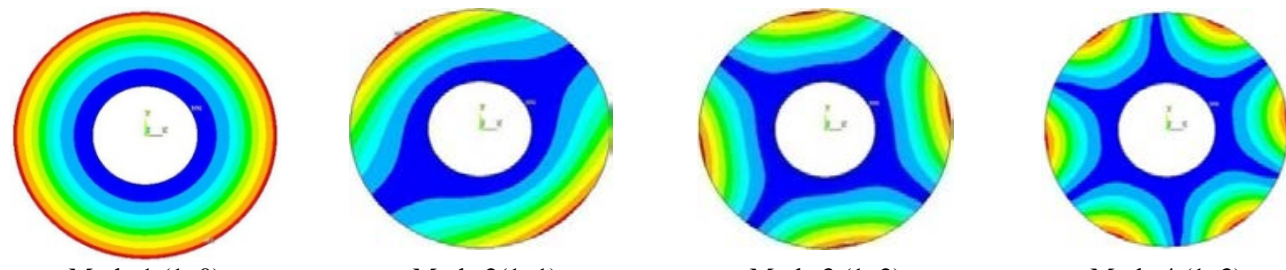

F-F
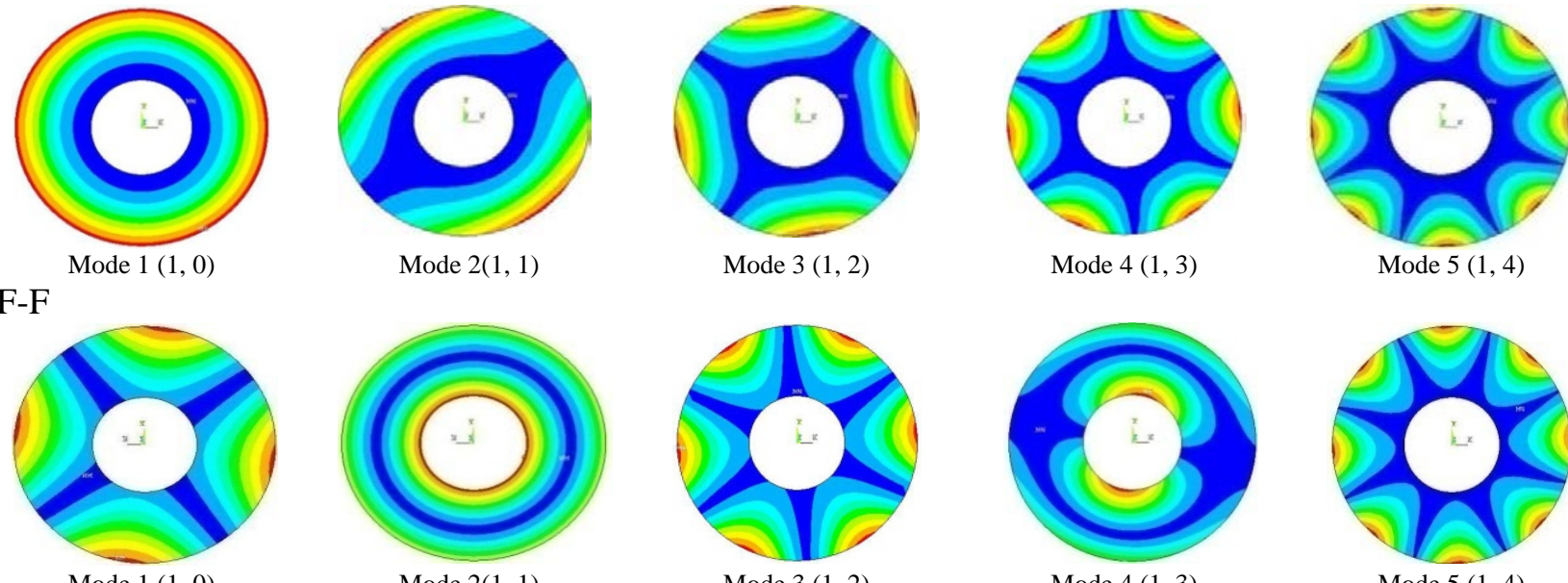

F-S
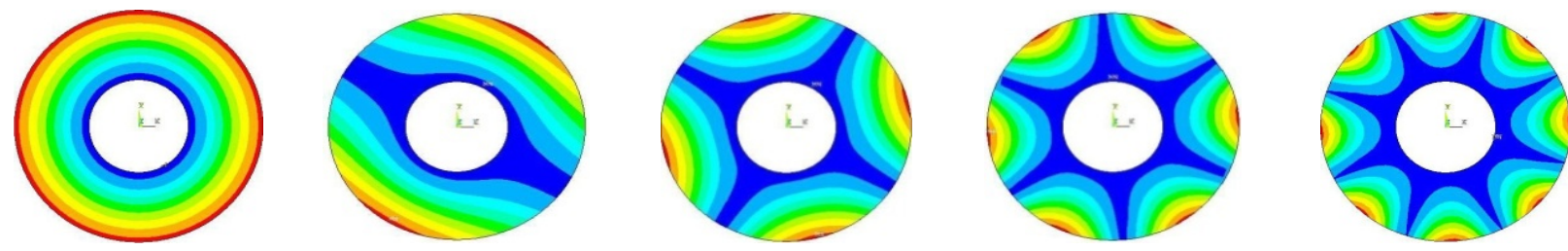

S-C

Mode 2(1, 1)

Mode 3 (1, 2)
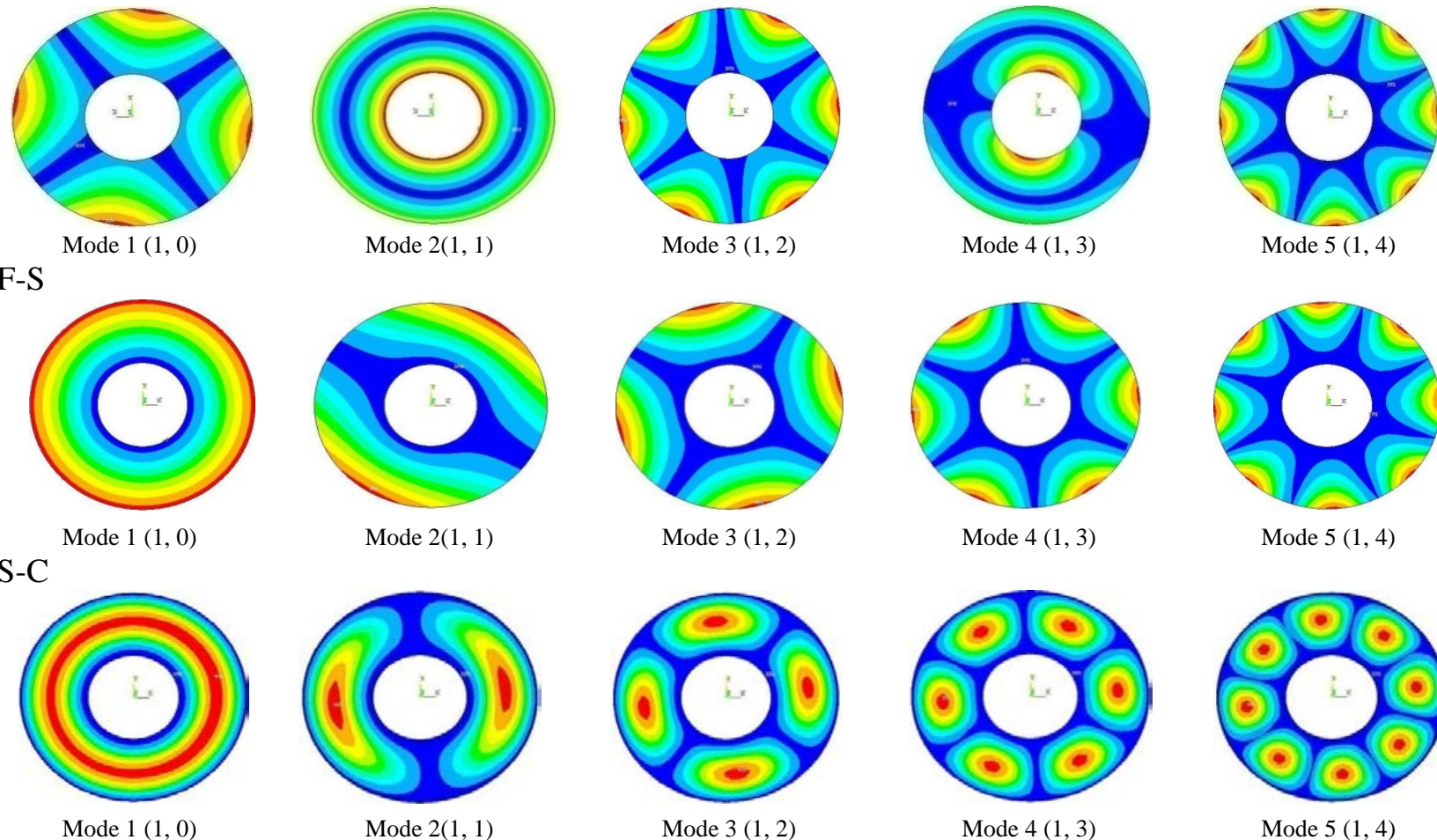

Mode $5(1,4)$

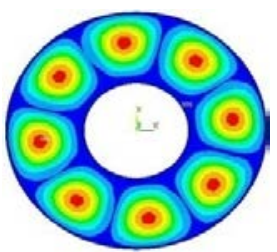

Mode $5(1,4)$ 


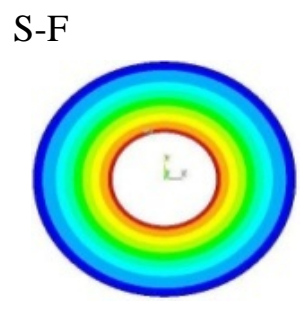

S-S

Mode $1(1,0)$

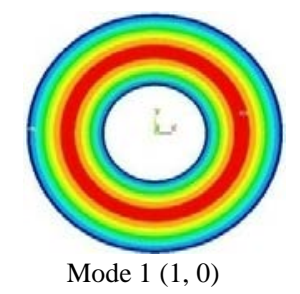

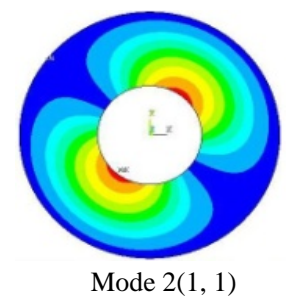

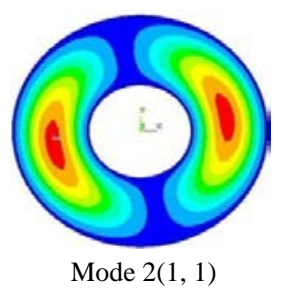

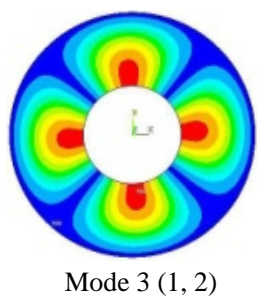

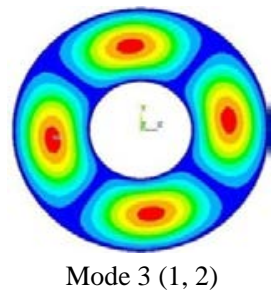

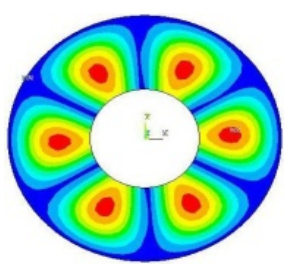

Mode 4 (1, 3)

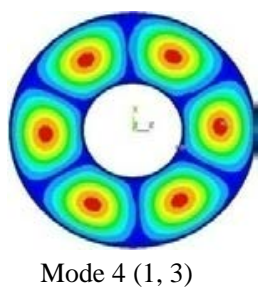

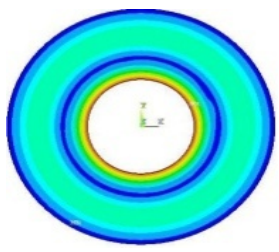

Mode $5(1,4)$

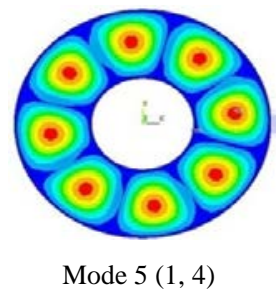

Fig. 5. The first five natural modes, for uniform annular plates with nine possible combinations of free, clamped and simply supported boundary conditions at the inner and outer edges of plates

\section{Conclusion}

In this paper, finite element method has been employed to solve free vibration of thin, isotropic circular and annular plates. The effects of boundary conditions and hole size on different modes of vibration has been fully investigated and it is found that the natural frequency increases as the hole size increases except when the inner and outer boundaries of the annular plates are free, for which they decrease with the hole size. It is also found that for free plate mode $(1,0)$ has higher frequency than mode $(1,2)$, as well as mode $(1,1)$ than $(1,3)$ in both circular and annular plates. The numerical results revel that the method is very accurate and can be extended to vibration problems of composite laminated plates which are subjects of investigation nowadays.

\section{References}

Bashmal, S., Bhat, R., \& Rakheja, S. (2009). In-plane free vibration of circular annular disks. Journal of Sound and Vibration, 322(1), 216-226.

Bashmal, S., Bhat, R., \& Rakheja, S. (2010). Frequency equations for the in-plane vibration of circular annular disks. Advances in Acoustics and Vibration, 2010.

Bhardwaj, H., Vimal, J., \& Sharma, A. (2015). Study of free vibration analysis of laminated composite plates with triangular cutouts. Engineering Solid Mechanics, 3(1), 43-50.

Chakraverty, S., \& Petyt, M. (1999). Free vibration analysis of elliptic and circular plates having rectangular orthotropy. Structural Engineering and Mechanics, 7(1), 53-67.

Chakraverty, S., Bhat, R. B., \& Stiharu, I. (2000). Vibration of annular elliptic orthotropic plates using two dimensional orthogonal polynomials. Applied Mechanics and Engineering, 5(4), 843-866.

Chakraverty, S., Bhat, R. B., \& Stiharu, I. (2001). Free vibration of annular elliptic plates using boundary characteristic orthogonal polynomials as shape functions in the Rayleigh-Ritz method. Journal of sound and vibration, 241(3), 524-539.

Chen, D. Y., \& Ren, B. S. (1998). Finite element analysis of the lateral vibration of thin annular and circular plates with variable thickness. Journal of vibration and acoustics, 120(3), 747-752.

Farag, N. H., \& Pan, J. (2003). Modal characteristics of in-plane vibration of circular plates clamped at the outer edge. The Journal of the Acoustical Society of America, 113(4), 1935-1946.

Kim, C. S. (2003). Natural frequencies of orthotropic, elliptical and circular plates. Journal of sound and vibration, 259(3), 733-745.

Komur, M. A., Sen, F., Ataş, A., \& Arslan, N. (2010). Buckling analysis of laminated composite plates with an elliptical/circular cutout using FEM. Advances in Engineering Software, 41(2), 161-164. 
Hassani, A., Hojjati, M. H., \& Fathi, A. R. (2010). In-plane free vibrations of annular elliptic and circular elastic plates of non-uniform thickness under classical boundary conditions. International Review of Mechanical Engineering, 4(1), 112-119.

Liang, B., Zhang, S. F., \& Chen, D. Y. (2007). Natural frequencies of circular annular plates with variable thickness by a new method. International journal of pressure vessels and piping, 84(5), 293-297.

Nia, M., Torabi, K., \& Heidari-Rarani, M. (2014). Free vibration analysis of a composite beam with single delamination-An improved free and constrained model. Engineering Solid Mechanics, 2(4), 313-320.

Reddy, J. N. (2004). Mechanics of laminated composite plates and shells: theory and analysis. CRC press.

Samaei, A. T., Aliha, M. R. M., \& Mirsayar, M. M. (2015). Frequency analysis of graphene sheet embedded in an elastic medium with consideration of small scale. Materials Physics and Mechanics, 22, 125-135.

Torabi, K., Afshari, H., \& Heidari-Rarani, M. (2013). Free vibration analysis of a non-uniform cantilever Timoshenko beam with multiple concentrated masses using DQEM. Engineering Solid Mechanics, 1(1), 9-20.

Vera, S. A., Sanchez, M. D., Laura, P. A. A., \& Vega, D. A. (1998). Transverse vibrations of circular, annular plates with several combinations of boundary conditions. Journal of Sound and Vibration, 213(4), 757-762.

Vera, S. A., Laura, P. A. A., \& Vega, D. A. (1999). Transverse vibrations of a free-free circular annular plate. Journal of Sound and Vibration, 224(2), 379-383.

Vimal, J., Srivastava, R., Bhatt, A., \& Sharma, A. (2014). Free vibration analysis of moderately thick functionally graded skew plates. Engineering Solid Mechanics, 2(3), 229-238.

Wang, C. Y. (2003). Vibration of an annular membrane attached to a free, rigid core. Journal of sound and vibration, 260(4), 776-782.

Wang, C. Y., \& Wang, C. M. (2005). Examination of the fundamental frequencies of annular plates with small core. Journal of sound and vibration, 280(3), 1116-1124.

Wu, T. Y., Wang, Y. Y., \& Liu, G. R. (2002). Free vibration analysis of circular plates using generalized differential quadrature rule. Computer Methods in Applied Mechanics and Engineering, 191(46), 5365-5380.

Yuan, J., \& Dickinson, S. M. (1996). On the vibration of annular, circular and sectorial plates with cutouts or on partial supports. Computers \& structures, 58(6), 1261-1264.

Yadav, D., Sharma, A., \& Shivhare, V. (2015). Free vibration analysis of isotropic plate with stiffeners using finite element method. Engineering Solid Mechanics, 3(3), 167-176.

Zhou, Z. H., Wong, K. W., Xu, X. S., \& Leung, A. Y. T. (2011). Natural vibration of circular and annular thin plates by Hamiltonian approach. Journal of Sound and Vibration, 330(5), 1005-1017. 